\title{
modélisation d'une cavité de stockage de gaz dans le sel considéré comme matériau élastoviscoplastique solution explicite
}

\author{
par \\ Nguyen Minh Duc \\ Pierre Berest
}

\section{Introduction}

On considérait initialement qu'il était inutile de décrire dans le détail les comportements différés dans le calcul des cavités de stockage dans le sel. Ses caractéristiques à long terme étaient supposées vérifier les lois de l'élastoplasticité parfaite (Salençon 1969) et étaient déduites d'expériences classiques en laboratoire.

Les multiples observations effectuées sur les cavités de stockage, attestant d'une réponse différée étalée sur plusieurs années (Berest 1981), ainsi que les expériences actuelles en laboratoire (Vouille 1981, Bergues 1981) mettent en évidence une viscosité du sel, bien plus importante que ne le laissait prévoir la schématisation initiale. De ce fait, les caractéristiques ultimes pouvaient être très largement surestimées; de plus, elles perdent beaucoup de leur intérêt pratique, puisqu'elles ne régissent le comportement des cavités qu'au bout d'un laps de temps, grand, par rapport à la période d'utilisation des cavités.

Le modèle élastoplastique "ultime» ne permet donc pas de comparer, par exemple, deux stratégies différentes d'utilisation du stockage définies par deux historiques différents de la pression intérieure. A cette fin, il faut, au contraire, utiliser un modèle qui décrive le comportement différé, dont le rôle reste essentiel pour les périodes de temps considérées (quelques années).

L'introduction de la viscoplasticité apparaît donc comme la deuxième étape des études consacrées aux cavités de stockage.

\section{Un modèle de calcul viscoplastique en symétrie sphérique}

Les modèles de calcul simplifiés, donnant lieu à des solutions explicites ou semi-explicites, constituent une première approche dans les tentatives actuelles de cerner le comportement in-situ des cavités de stockage et sont un instrument particulièrement souple pour la modélisation; ils permettent accessoirement de tester les codes de calculs sur les gros ordinateurs.
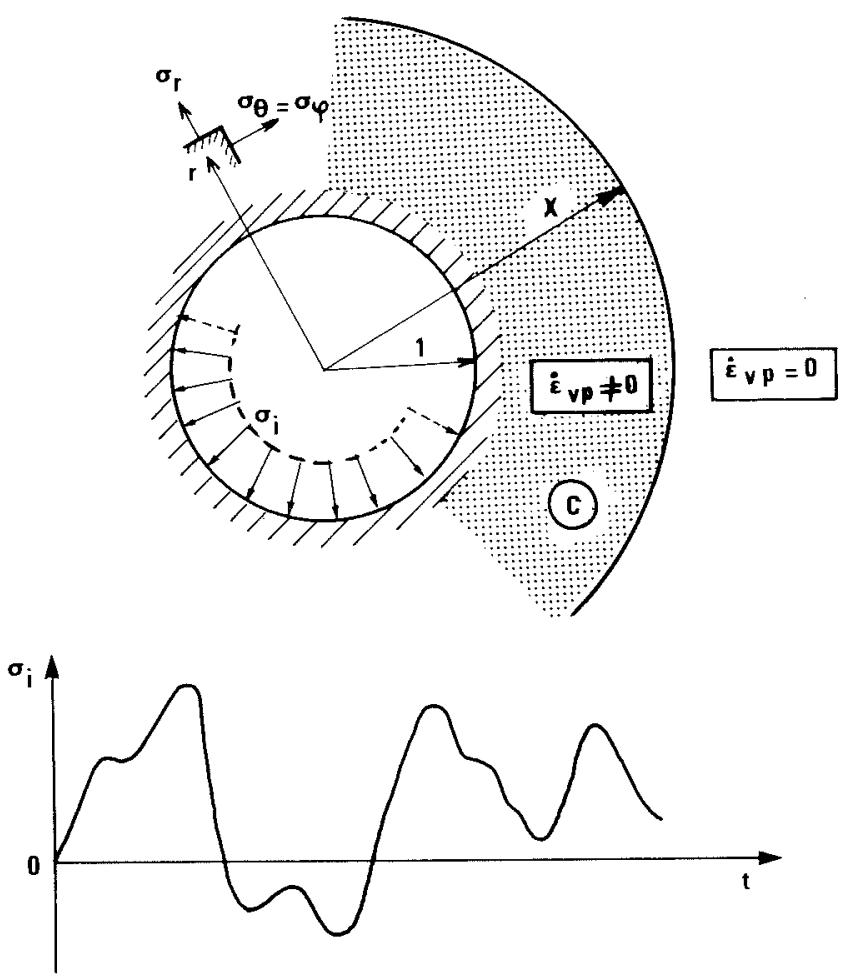

Fig. 1 Modèle géométrique

\subsection{Géométrie (fig. 1)}

On considère une cavité sphérique en milieu infini, soumise sur sa paroi à une contrainte normale uniforme variant suivant un historique quelconque : $\sigma_{i}=\sigma_{i}(t)$

Le comportement du sel étant supposé indépendant de la pression moyenne, $\sigma_{i}$ représente en fait, la différence entre la pression géostatique au niveau de la cavité et la pression à l'intérieur de la cavité.

Les notations en symétrie sphérique sont rappelées sur la figure 1 . On désignera par $r=x$ le rayon, variable, de la zone en charge plastique entourant la cavité. 


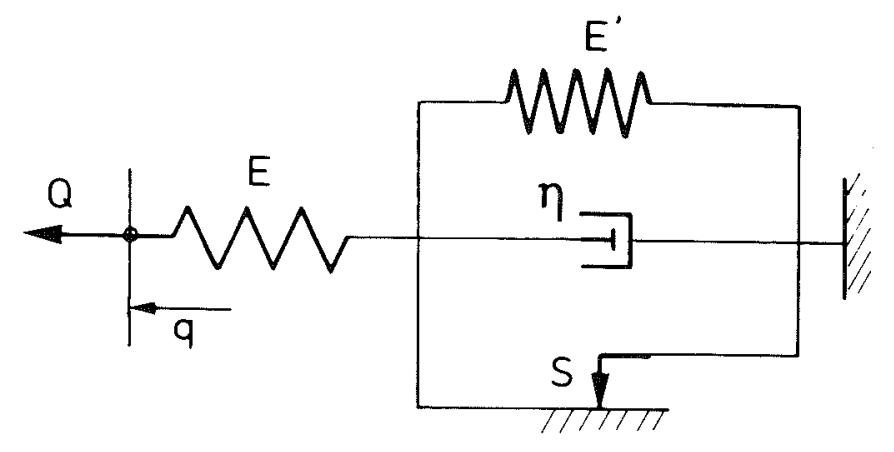

Fig. 2 Modèle rhéologique

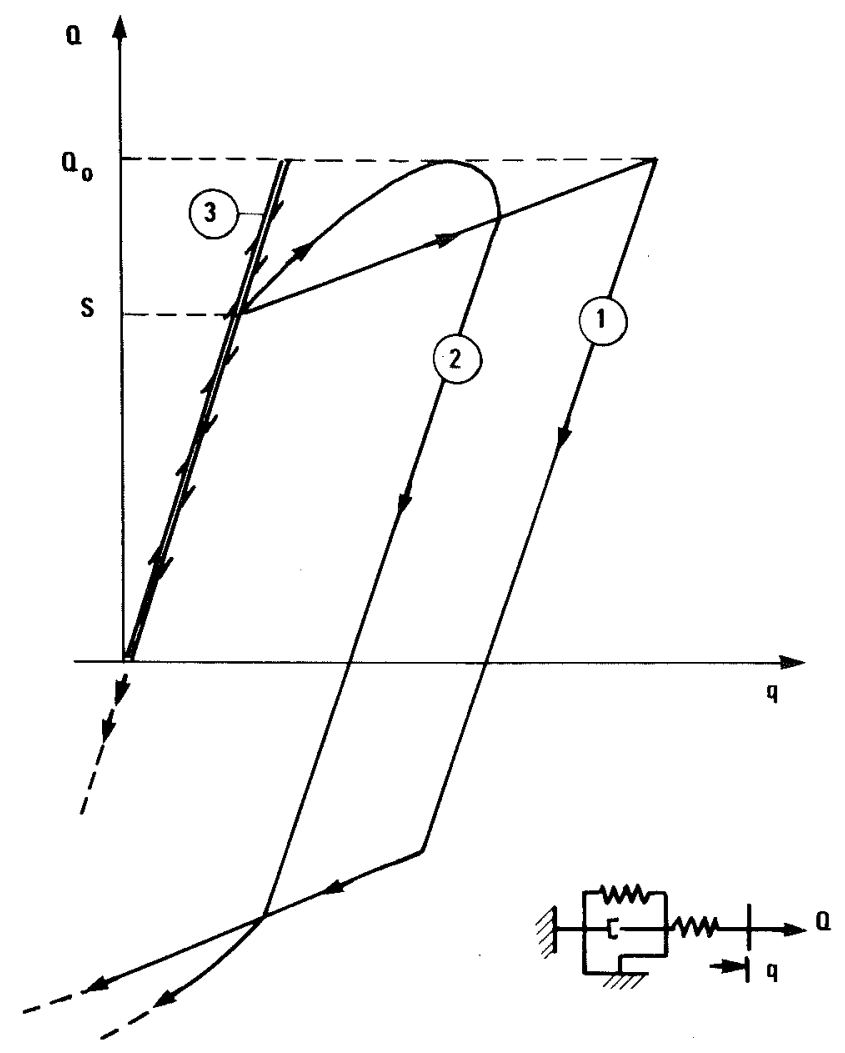
Fig. 3 Réponse du modèle rhéologique
(1) élastoplasticité : $\eta \rightarrow 0$ ou $\dot{Q} \rightarrow 0$
(2) cas général
(3) "élasticité»: $\eta \rightarrow \infty$ ou $\dot{Q} \rightarrow \infty$

\subsection{Modèle de comportement viscoplastique}

Le modèle rhéologique est représenté sur la figure 2 .

II s'agit d'un matériau de Bingham, avec écrouissage cinématique (qui peut être annulé : $E^{\prime}=0$ ).

Rappelons la réponse de ce modèle, soumis à une force $Q$ croissante jusqu'à $Q_{0}$ (suffisamment grand), puis décroissante, $\eta$ étant la constante de viscosité et $\dot{Q}_{0}$ la vitesse de chargement (fig. 3).
a) $\eta \longrightarrow 0$
ou
$\dot{\mathrm{Q}} \longrightarrow 0$ :
réponse
élastoplastique.

b) $\eta \longrightarrow \infty$ ou $\dot{\mathrm{Q}} \longrightarrow \infty$ : réponse «élastique».

c) Cas général : la réponse est «intermédiaire» et dépend de $\eta$ et $\dot{Q}$.

\section{3 Équations et résolution du problème}

\section{1 Équations}

L'équation d'équilibre, la décomposition de la déformation en partie élastique et viscoplastique, la loi d'évolution de la déformation viscoplastique, fournissent les équations du problème:

$$
\begin{gathered}
\frac{\partial \sigma_{r}}{\partial r}+\frac{2}{r}\left(\sigma_{r}-\sigma_{\theta}\right)=0, \\
E \frac{\partial u}{\partial r}=\sigma_{r}-2 \nu \sigma_{\theta}+E \varepsilon_{v p}, \\
E \frac{u}{r}=\sigma_{\theta}(1-\nu)-v \sigma_{r}-E \frac{\varepsilon_{v p}}{2}, \\
\dot{\varepsilon}_{\mathrm{vp}}=\frac{1}{\eta}<|\Psi|-S>\operatorname{sgn} \Psi, \\
\Psi=\sigma_{r}-\sigma_{\theta}-E^{\prime} \varepsilon_{\mathrm{vp}} .
\end{gathered}
$$

\section{Remarques:}

- La variation volumique viscoplastique est nulle. - $\varepsilon_{v p}$ est la déformation viscoplastique radiale.

- $S$ est le seuil initial d'écoulement du matériau en chargement uniaxial.

- Dans (4), $\langle\mathbf{f}\rangle$ signifie «valeur positive de $f »$ :

$$
\left\{\begin{array}{l}
=f \text { si } f>0 \\
=0 \text { si } f<0
\end{array}\right.
$$

- $|\Psi|-S=0$ définit la frontière d'écoulement viscoplastique.

- Enfin, sgn $\Psi$ rend compte du chargement plastique «dans un sens » $\left(\dot{\varepsilon}_{\mathrm{vp}}>0\right)$ ou «dans l'autre $\left(\dot{\varepsilon}_{\mathrm{vp}}<0\right)$.

\subsection{Résolution}

La résolution du problème passe par la détermination de la frontière $r=x(t)$ de la zone en charge viscoplastique $\left(\dot{\varepsilon}_{\mathrm{vp}} \neq 0\right)$, entourant la cavité et qu'on notera zone $(\mathrm{C})$.

La figure 4 donne un exemple d'évolution de $x$ en fonction du temps $t$. Ainsi, la partie $\widehat{A B C D E}$ de la courbe $x(t)$, est liée aux développements suivants de la zone (C) :

- Phase I) $\widehat{A B}$ : apparition, puis développement de (C) dans le milieu à l'état initial naturel.

- Phase II) $\overparen{B C}$ : régression de la zone (C) $(\dot{x}<0)$, laissant derrière elle des déformations permanentes.

- Phase III) $\widehat{C D}$ : expansion à nouveau de (C), dans un milieu déjà plastifié.

- Phase IV) $\overparen{D E}$ : la zone (C) débouche à nouveau dans un milieu à l'état naturel.

Remarquons qu'au cours de l'évolution, une phase II peut se terminer par la disparition totale de (C); il lui succède une phase élastique, qu'on représente conventionnellement sur la figure 4 par un segment de droite $x=1$ : $F G, I J, O P \ldots$

La phase III apparaît ensuite, soit avec $\dot{\varepsilon}_{\mathrm{vp}}$ de même signe que dans la phase II précédente, soit de signe contraire. Les équations régissant ces différentes phases sont données en annexe. 


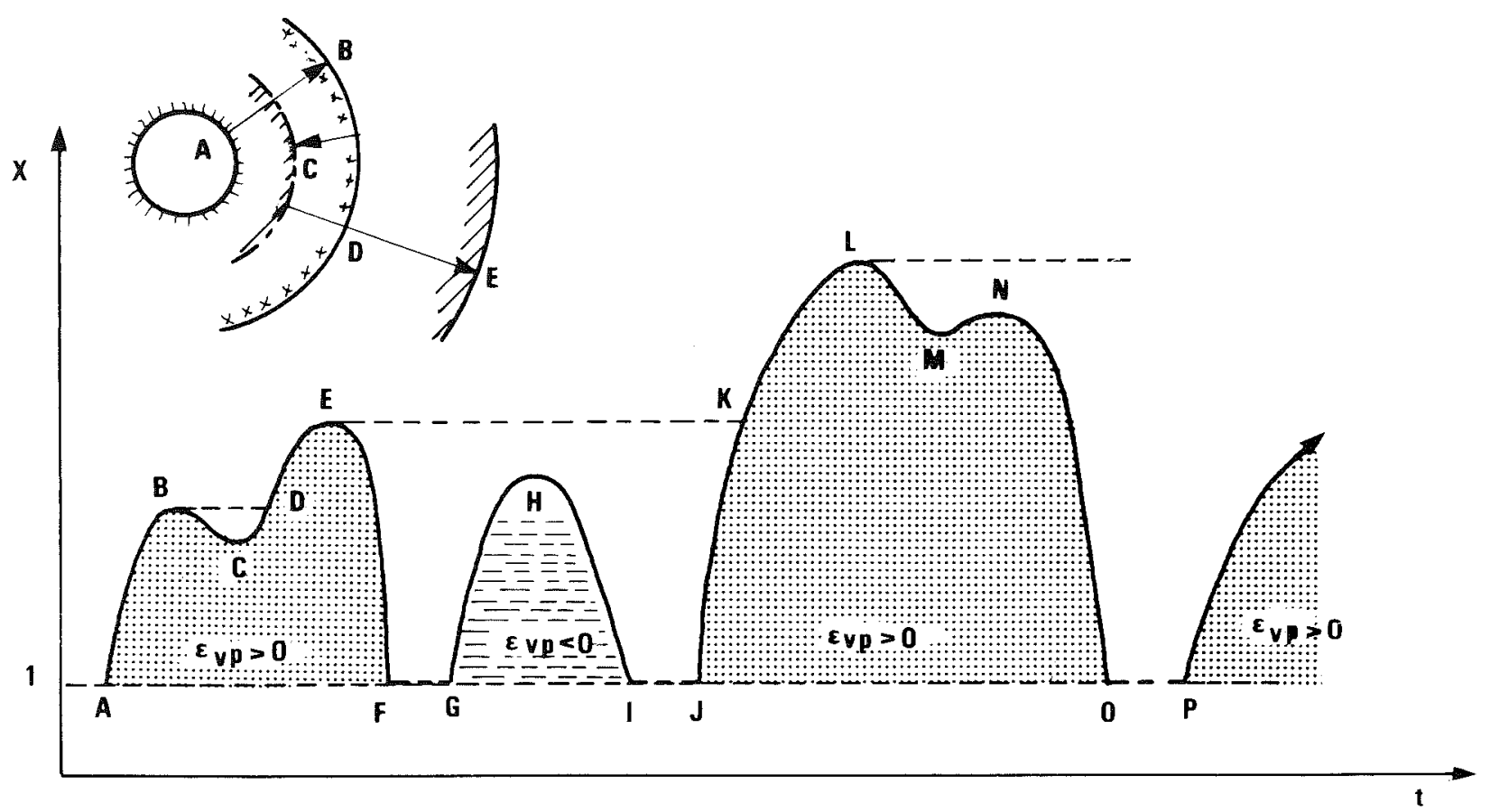

Fig. 4 Exemple d'évolution de la zone en charge viscoplastique
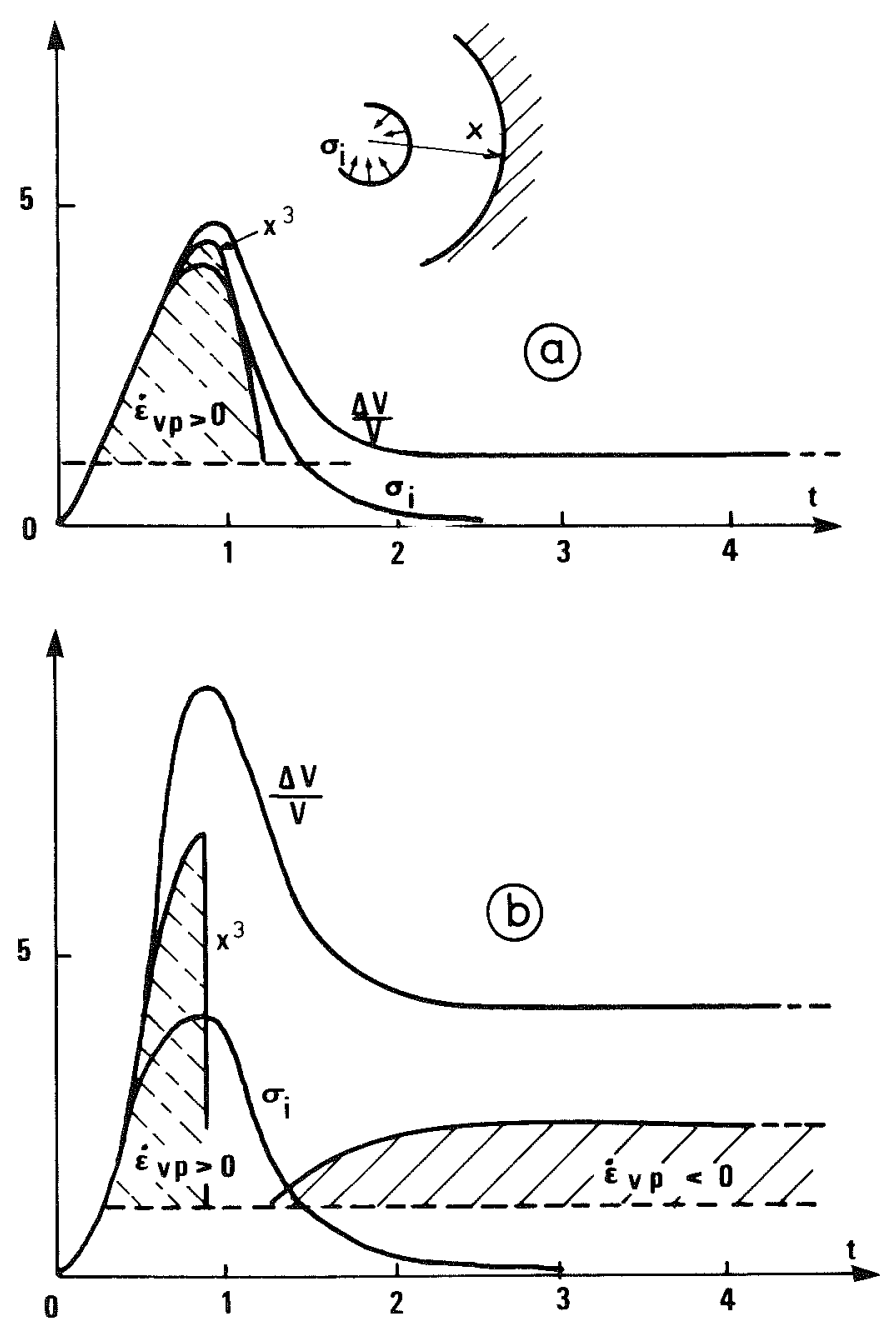

Fig. 5 Chargement de type «impulsion»:

a) $\tau=1$

b) $\tau=0.006$

\section{Exemples de chargement}

On adopte la présentation suivante: on porte sur la même figure, en fonction du temps,
- la contrainte appliquée $\sigma_{i}(t)$,

- le cube du rayon plastique $x^{3}$,

- la variation volumique de la cavité $\frac{\Delta V}{V}$.

Les courbes représentatives passent toutes par le même point initial d'ordonnée 1, les paramètres étant rapportés à leurs valeurs respectives à l'apparition de la plasticité.

\subsection{Choix d'une «constante de temps » $(\tau)$}

Il est difficile de définir une constante de temps de réponse de la structure pour un phénomène qui dépend de la viscosité du matériau et du chargement. Pour une sollicitation donnée, on pourra cependant caractériser une "certaine» réponse par la constante $\tau=\alpha^{-1}$ qui apparaît dans les formules (6) à (8) et dont on reviendra plus loin sur la signification:

$$
\tau=2(1-v) \eta \cdot\left(E+2(1-v) E^{\prime}\right)^{-1} \text {. }
$$

Ainsi :

- $\tau \longrightarrow \infty$ les trois courbes sont confondues; on retrouve bien la réponse élastique, puisque la variation du volume est proportionnelle à la sollicitation $\sigma_{i}(t)$. La proportionnalité de $x^{3}$ et $\sigma_{i}(t)$, pour $\sigma_{i}(t)>1$, montre qu'il y a développement d'une zone de charge viscoplastique, bien que cette zone n'ait pas le temps de créer des déformations permanentes.

- $\tau \longrightarrow 0:$ on retrouve la réponse élastoplastique : la disparition de la zone plastique (décharge) ou sa réapparition (charge) se fait de façon instantanée.

Choisissons, pour fixer les idées, l'année comme unité de temps. Dans les exemples qui vont suivre, pour ces cycles de l'ordre de l'année, on pourra apprécier le rapprochement du comportement de la cavité de l'un ou de l'autre cas extrême précédent.

\subsection{Chargement de type «impulsion» (fig. 5)}

Cette figure illustre deux comportements nettement différenciés, pour des constantes $\tau=1$ an et $\tau=$ 2,2 jours (fig. 5 a et 5 b). Le comportement de type 


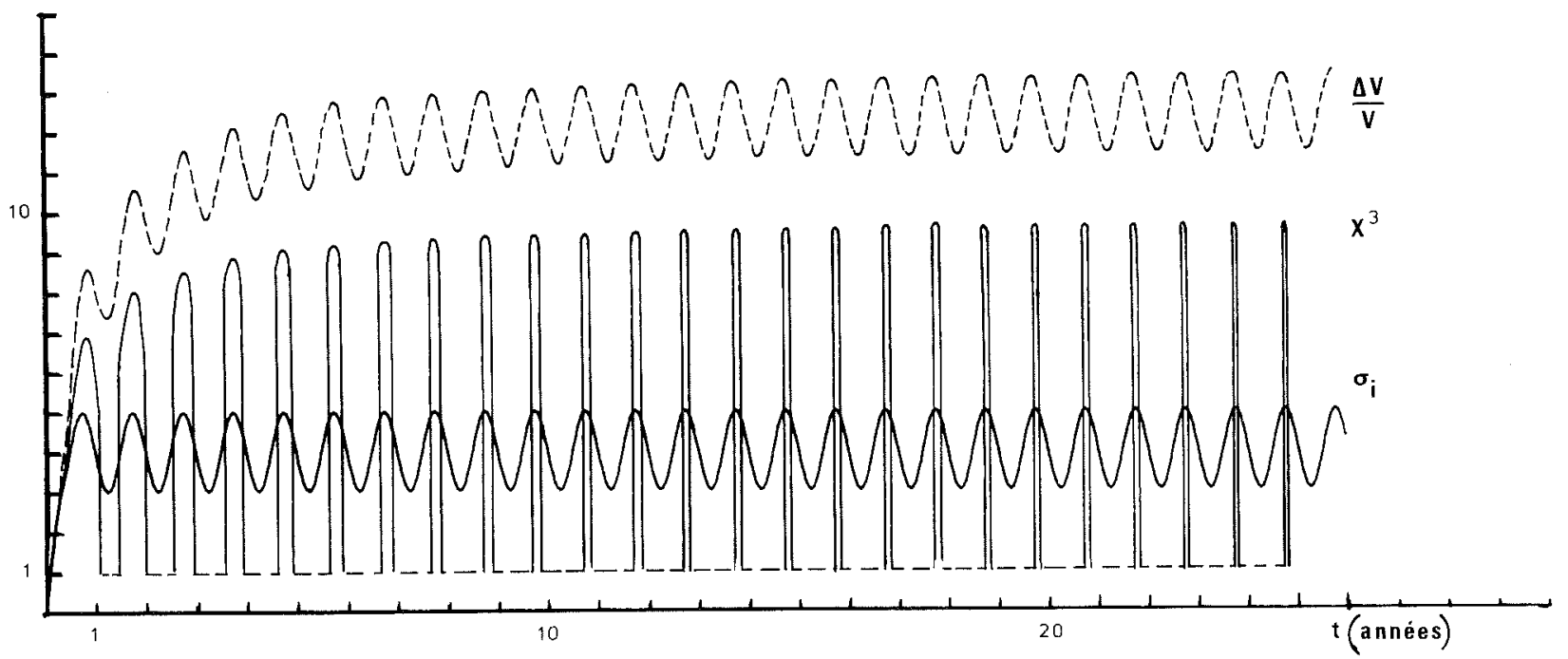

Fig. 6 Chargement cyclique d'intensité moyenne $\tau=0.25$

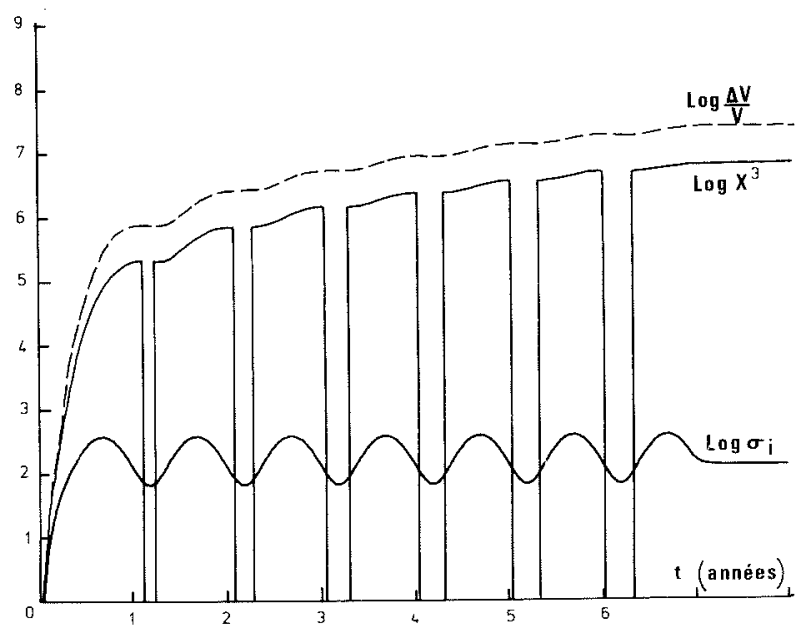

élastoplastique » est bien visible, dans ce dernier cas, avec une décharge quasi instantanée et la réapparition, avec stabilisation, d'une nouvelle zone en charge plastique "dans l'autre sens». II est cependant important de noter que, d'un point de vue quantitatif, on reste encore loin de l'élastoplasticité $(\eta=0)$ : la valeur maximale de $x^{3}$ est ici de 7 contre $x^{3}=9,4$ pour $\eta=0$.

\subsection{Chargement cyclique (fig. 6 et 7 )}

Les fonctions $\sigma_{i}(t)$ schématisent ici grossièrement le chargement des cavités de stockage en gaz. Les exemples suivants illustrent l'influence de l'intensité du chargement sur l'étalement du phénomène global, qui se superpose à l'effet «local» du paramètre $\tau$.

a) Chargement «moyen» (fig. 6)

La constante de temps $\tau$ est de 4 mois $(E=24000 \mathrm{MPa}$, $\left.\mathrm{E}^{\prime}=6000 \mathrm{MPa}\right)$ :

$$
\sigma_{i}(\max )=5 ; \quad \sigma_{i}(\min )=4 .
$$

Fig. 7 Chargement cyclique de forte intensité $\tau=0.02$

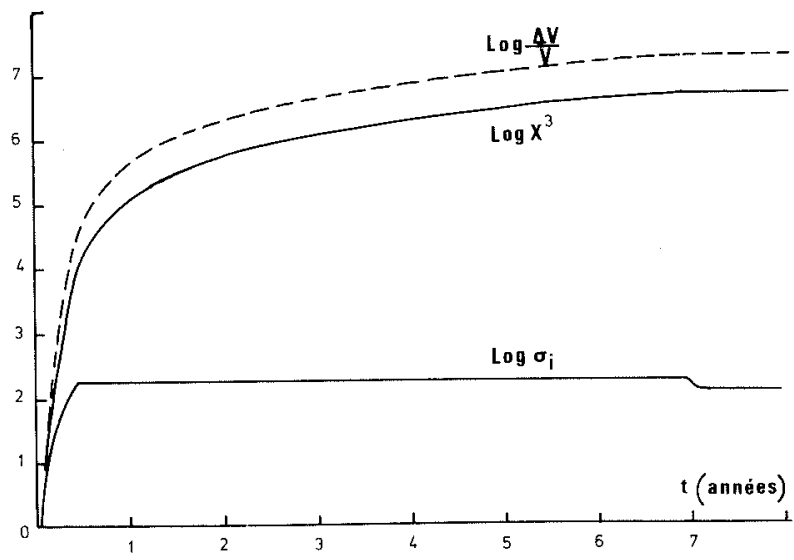

Fig. 8 Chargement "moyen» du chargement dans la fig. 7 , tous les autres paramètres étant conservés

II y a une succession de phases plastiques et élastiques, avec une tendance à l'adaptation. L'évolution vers l'adaptation est très lente. Par contre, $80 \%$ de la perte de volume est déjà acquise au bout de deux ans.

b) Chargement «important» (fig. 7)

$\sigma_{i}(\max )=13, \quad \sigma_{i}(\min )=6:$ l'importance des déformations (plus de $35 \%$ en 7 ans) nous ont conduit à utiliser des coordonnées semi-logarithmiques. Cet exemple pourrait représenter les conditions de Tersanne, si on adoptait les caractéristiques suivantes, relativement pessimistes:

- cohésion 1,5 MPa (ou seuil $3 \mathrm{MPa}$ ),

- module d'Young $20000 \mathrm{MPa}$,

- écrouissage nul,

- viscosité $\eta=270 \mathrm{MPa} \cdot$ an;

- pressions extrêmes dans la cavité :

$$
\mathrm{P}_{\mathrm{i}} \max =22 \mathrm{MPa}, \quad \mathrm{P}_{\mathrm{i}} \min =8 \mathrm{MPa} \text {, }
$$

- pression géostatique : $34 \mathrm{MPa}(=-1500 \mathrm{~m})$.

Malgré une constante de temps plus faible que dans le cas précédent, $(\tau=1$ semaine), ce qui conduit à des décharges et recharges plastiques quasi instantanées, le phénomène global s'étend sur une période de plusieurs années. Ainsi, pour comparer avec l'exemple précédent, la perte de volume, au bout de la $2^{\mathrm{e}}$ année 
n'est que de $35 \%$ de celle obtenue au bout de sept ans, date à laquelle la stabilisation n'est pas achevée. En effet, on a alors $\log x^{3}=6,9$ contre $\log x^{3}=12$ dans le cas $\eta=0$; ces valeurs ne sont en fait qu'indicatives, puisqu'on sort alors du domaine des petites déformations.

Remarque: L'étalement du phénomène se reflète mal sur la figure, du fait des coordonnées semilogarithmiques qu'on a dû utiliser.

\subsection{Discussion des résultats}

Les exemples font apparaître deux constantes de temps différentes.

- La constante $\tau$ est analogue à une constante de relaxation. En faisant $\dot{x}=0$ dans l'équation (6), ce qui revient à bloquer les déformations volumiques plastiques, $\tau$ définit la relaxation de $\sigma_{i}(\mathrm{t})$. II caractérise également les réponses de décharge et recharge, d'après les formules (7) et (8) en annexe.

- L'évolution "générale" semblerait plutôt suivre une constante de temps de fluage, plus grande que $\tau$ et d'autant plus, que le chargement relatif est important. Sur la figure 8 , on retrouve bien une même tendance que dans l'exemple de la figure 7 , en remplaçant le chargement cyclique par sa moyenne.

La constante de fluage de la cavité peut être définie en faisant $\dot{\sigma}_{\mathrm{i}}=0$ dans la formule (6) en annexe; son expression, sous forme d'intégrale, est plus complexe que celle de $\tau$; mais l'écart par rapport à ce comportement «moyen » ne peut être apprécié que par l'étude directe du comportement sous les sollicitations variables.

\section{Conclusion}

Une discussion complète du modèle pour différents schémas d'exploitation n'a pas encore été faite. Les quelques résultats obtenus jusqu'ici illustrent cependant bien la souplesse d'emploi des modèles de calcul simplifiés en vue de la modélisation du comportement in-situ.

En particulier, ce modèle, avec un petit aménagement, pourrait être utilisé à l'interprétation d'une expérience du type de celle de Kiel (Kühne et al., 1973); dans un premier temps, on vide la colonne de saumure dans le tube au-dessus de la cavité, ce qui ramène «instantanément » la pression à zéro, au toit de la cavité. Dans un deuxième temps, on enregistre la remontée de la colonne de saumure en fonction du temps, du fait du fluage de la cavité et du réchauffement de la saumure : la contrainte $\sigma_{i}$ est ici une fonction de la variation volumique.

\section{Annexe}

Équations régissant l'évolution de la zone en charge viscoplastique (C), de rayon $x$ (se reporter au paragraphe 3.2 )

$1^{\circ}$ Phase I

(6) $\alpha \sigma_{i}+\dot{\sigma}_{i}=2 S / 3 \operatorname{sgn} \Psi\left(x^{3}+E^{\prime} \eta^{-1} x^{3}\right.$

$$
\left.+E(2 \eta(1-v))^{-1} \cdot\left(1+\log x^{3}\right)\right),
$$

avec

$$
\alpha=\eta^{-1}\left(E^{\prime}+E(2(1-v))^{-1}\right) \text {. }
$$

$2^{\circ}$ Phases // et III - Les valeurs actuelles de $\dot{x}\left(t=t_{n}\right)$ sont des fonctions explicites de quantités connues lors du dernier passage de la frontière de (C) au point $r=x\left(t=t_{n-1}\right)$. On désigne par e la valeur maximale atteinte par $x(t)$ et $\sigma_{e}$, la valeur de $\sigma_{r}$ en ce point.

a) Phase II:

(7) $\dot{\overline{x^{3}}}\left(t_{n}\right)=\dot{\mathrm{x}^{3}}\left(t_{n \cdots 1}\right)[1$

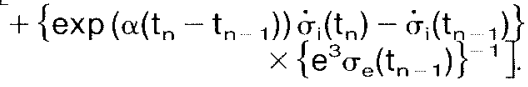

b) Phase III:

$$
\begin{aligned}
& \hat{x^{3}}\left(t_{n}\right)=\left\{\widehat{e^{3} \sigma_{e}}\left(t_{n \cdots 1}\right)+\left[\alpha \sigma_{i}(t)+\dot{\sigma}_{i}(t)\right]_{t_{n}^{n}}^{t}\right. \\
& \left.-\frac{2 S}{3}[\operatorname{sgn} \Psi(t)]_{t_{n}^{n}}^{n}, h\left(x^{3}\right)\right\} \\
& \left.\times\left\{\widehat{\left(\mathrm{e}^{3} \sigma_{e}\right.}\left(t_{n-1}\right)\right) / \dot{x^{3}}\left(t_{n}-1\right)+\frac{2 S}{3}[\operatorname{sgn} \Psi(t)]_{n_{n-1}^{n}}^{t}\right\}
\end{aligned}
$$

en notant:

$h\left(x^{3}\right)=E^{\prime} \eta^{-1} x^{3}+E(2 \eta(1-v)){ }^{1}\left(1+\log x^{3}\right)$.

$3^{\circ}$ Phase $N$ - Cette phase est régie par la même équation (6).

\section{Références bibliographiques}

P. BEREST, (1981), Stabilité des cavités de stockage d'hydrocarbures dans le sel; données de l'expérience internationale. Revue Française de Géotechnique, dans ce même numéro

J. BERGUES, (1981), Comportement rhéologique du sel. Revue Française de Géotechnique, (à paraître).

KUHNE, ROHR, SASSE, (1973), "Stockage du gaz à Kiel». $12^{\mathrm{e}}$ Congrès Mondial du Gaz, Nice 1973.

J. SALENÇON, (1969), Contraction quasi-statique d'une cavité à symétrie sphérique ou cylindrique dans un milieu élastoplastique. Annales des Ponts et Chaussées, vol. IV, pp. 231-236.

P. M. STREMDORFER, (1972), Résolution par la méthode des éléments finis des problèmes viscoélasti ques et élastoplastiques posés par les cavités souterraines. Thèse Docteur-Ingénieur, 9 juin 1972, Université Paris 6.

G. VOUILLE, (1981), Revue Française de Géotechnique, (à paraître). 